University of Nebraska - Lincoln

DigitalCommons@University of Nebraska - Lincoln

PreColumbian Textile Conference VIII /

Jornadas de Textiles PreColombinos VIII (2019)

Centre for Textile Research

$6-2020$

\title{
Una prenda triangular con plumas en la colección del Museo de sitio de Pachacámac
}

Lourdes Chocano Mena

Follow this and additional works at: https://digitalcommons.unl.edu/pctviii

Part of the Art and Materials Conservation Commons, Fiber, Textile, and Weaving Arts Commons, Indigenous Studies Commons, Latin American Languages and Societies Commons, Museum Studies Commons, and the Other History of Art, Architecture, and Archaeology Commons

This Article is brought to you for free and open access by the Centre for Textile Research at DigitalCommons@University of Nebraska - Lincoln. It has been accepted for inclusion in PreColumbian Textile Conference VIII / Jornadas de Textiles PreColombinos VIII (2019) by an authorized administrator of DigitalCommons@University of Nebraska - Lincoln. 


\title{
Una prenda triangular con plumas en la colección del Museo de sitio de Pachacámac
}

\author{
Lourdes Chocano Mena
}

\begin{abstract}
Resumen
Las formas habituales de los tejidos son la cuadrangular o la rectangular. Sin embargo, existen casos de tejidos como los paños trapezoidales de algodón usados como prendas de vestir (taparrabos, turbantes, pañuelos). Aquí presentamos un estudio de una prenda de forma triangular hallada en Pachacámac. Su hallazgo no recibió mayor atención, pese a su gran dimensión y la manera de cómo ha sido confeccionada, es muy particular: sus bordes están recamados de plumas anaranjadas y moradas en hileras. Explicaremos la confección de cada una de sus partes, haremos comparaciones con otras vestimentas de forma similar, y propondremos las posibles funciones que pudo tener este tejido triangular con plumas en su contexto.
\end{abstract}

Palabras claves: Textiles andinos, Pachacámac, prenda triangular, textil plumario.

\begin{abstract}
The most frequent shape of textiles is either quadrangular or rectangular. However, there are cotton textiles in trapezoidal form worn as personal attire (loincloths, scarves, turbans). We present here a study of a piece in triangular shape from Pachacámac, which has not been analyzed before. Its dimension and its manufacture are peculiar: its hems were decorated with orange and purple feathers in rows. We will explain the making of each section, compare it with other triangular garments, and illustrate the possible functions of such a textile in its context.
\end{abstract}

Keywords: Andean textiles, Pachacámac, triangular garment, feathered textile.

\section{Résumé}

Les formes habituelles des tissus sont quadrangulaires ou rectangulaires. Cependant, il existe des tissus en coton de forme trapézoïdale utilisés comme vêtements (pagnes, turbans, étoffes). Nous présentons ici une étude d'un tissu de forme triangulaire découvert à Pachacamac qui n'a pas été analysé auparavant. Ses dimensions et la manière dont il a été confectionné sont particulières : ses bords ont été décorés de plumes orangées et mauves disposées en bandes. Nous expliquons l'élaboration de chacune de ses parties, en le comparant à d'autres formes de vêtements similaires, et nous proposons les possibles fonctions qu'a pu avoir ce tissu triangulaire à plumes selon son contexte.

Mots-clés: Textiles andins, Pachacamac, vêtement triangulaire, tissus à plumes. 


\section{Antecedentes}

Los tejidos andinos se han caracterizado por la constancia de su forma, siempre son paños rectangulares o cuadrangulares, los cuales algunas veces se juntan para obtener mayor dimensión. Esto sucede especialmente cuando los tejidos son elaborados en telares de cintura, porque los paños producidos por lo general tienen una anchura limitada, un máximo de unos $85 \mathrm{~cm}$. (Desrosiers 1992: 11); en estos casos lo común era unir dos o más paños hasta conseguir el tamaño deseado. Sin embargo, con los telares horizontales o verticales existe la posibilidad de obtener tejidos de mayor dimensión, por tener una trama más ancha.

No obstante, se presentan algunas variaciones formales, aunque no son frecuentes. Así se hallan textiles prehispánicos producidos en telar con formas diferentes, por ejemplo: paños ligeramente angostos en el centro y que se expanden en los bordes superior o inferior (hipérbolas); es el caso de algunos taparrabos procedentes de Pachacámac, así como los paños triangulares (VanStan, 1965, 1967), los paños trapezoidales de bolsas o chuspas (Huaca Malena), así como los paños que conforman algunos uncus (Agüero, 1998: 103, 128 Imágenes 122, 123). Asimismo hay tejidos de formas casi triangulares como ciertos taparrabos de Paracas (Carrión Cachot, 1931: 82,83 (fig. 20 E), y también textiles de forma definidamente triangular como algunos especímenes en la costa norte y central (Reiss y Stübel, $1880-1887$, vol. II: 146, 147 lámina 43), además de textiles en forma de triángulos escalonados confeccionados mediante la técnica de tapiz (Rowe, 1999: 445, lámina 20.) Finalmente, existen textiles de formas ovaladas, triangulares, oblongas realizados con técnicas sin telar.

\section{El tejido triangular de bordes recamados con plumas de Pachacámac ${ }^{1}$}

Teniendo como antecedente lo mencionado, estudiaremos un tejido de forma triangular cuya característica es la manera en que se ha elaborado para lograr esa forma: un triángulo de tipo obtuso, es decir, de dos ángulos agudos en los extremos y en el centro uno de más de $90^{\circ}$ grados. Además presenta una peculiar decoración de un recamado de plumas anaranjadas y azulado tornasol en dos de sus bordes (foto 1). Finalmente, plantearemos una serie de comparaciones y posibilidades y de qué manera pudo utilizarse.

Este tejido proviene del área de Pachacámac, el cual es conocido como uno de los más importantes santuarios de la época inca, ubicado estratégicamente a escasos metros del litoral. Su ocupación y fama data desde tiempos anteriores al imperio del Tawantinsuyo, como han demostrado los numerosos estudios y excavaciones realizados en este lugar, quedando en claro que hubo gran dinamismo e interrelación social entre los habitantes que residían y trabajaban en el mismo santuario, y aquellos peregrinos que iban a ofrendar al dios del santuario (Eeckhout, 2017).

El tejido que estudiamos fue hallado en el área conocida como la "Portada de la costa" formada por las murallas segunda y tercera del santuario, justamente donde fueron seccionadas para dar paso a la vía llamada antigua Panamericana (Ramos 2012: 179, fig. 76).

En retrospectiva la "Portada de la costa" fue intervenida tres veces, la primera vez en 1994/95 por los arqueólogos Daniel Guerrero y Hernán Carrillo, en esa oportunidad se encontraron fragmentos de mullus y figurinas de arcilla, los cuales se dataron en el periodo Inca. También se encontró un entierro de una mujer joven, orientada de forma paralela al eje del muro, sin embargo solo tenía un envoltorio de tejido llano de algodón y no presentaba ninguna otra ofrenda. Luego, en otro momento del mismo proyecto se hallaron recintos rectangulares sobre un muro, los que fueron utilizados en la época inca, y contenían abundante material orgánico, moluscos y diez porras circulares. Los recintos habían sido sellados, una vez depositados los objetos; por eso los arqueólogos plantearon que se trataba de ofrendas (Ramos, 2012).

En 1996, esta área vuelve a ser excavada por los arqueólogos Rocío Aramburu y Marco Machaguay (Ramos, 2012), quienes encontraron más recintos, algunos de los cuales estaba asociado a un basural con restos de actividades artesanales o de producción; también un sector mostraba fases de ocupación desde el período final de Yschma y la última ocupación Inca, igualmente con materiales culturales de esos estilos. Asimismo se identificaron vestigios de estilos foráneos como Chancay y Chimú. Ante estas evidencias, los autores plantearon que el abundante material correspondía a talleres de producción alfarera, a pesar de que había algunos textiles, sobre los cuales no se hicieron mayores propuestas o estudios en ese momento. Entre dichos tejidos se encontraba el paño triangular con plumas en que se centra el presente artículo.

Antes de continuar con la descripción del paño, daremos una visión general del tipo de colección textil recuperada procedente del Santuario de Pachacámac. Existen especímenes recolectados desde el siglo VII al XVI (6oo al 1500 d. C) correspondientes a los estilos Huari, Lambayeque, Yschma e Inca (Feltham y Ángeles 2017). Los tejidos de estilos tardíos

1. Este textil fue identificado en el transcurso de las labores de conservación textil de 2009 en el Museo de Sitio de Pachacamac. La autora agradece a dicha entidad, a su directora Dra. Denise Pozzi-Escot, a la responsable del gabinete textil Sra. María Luisa Patrón y a la encargada del área de Manejo de Colecciones, Lic. Sonia Quiroz Calle. 


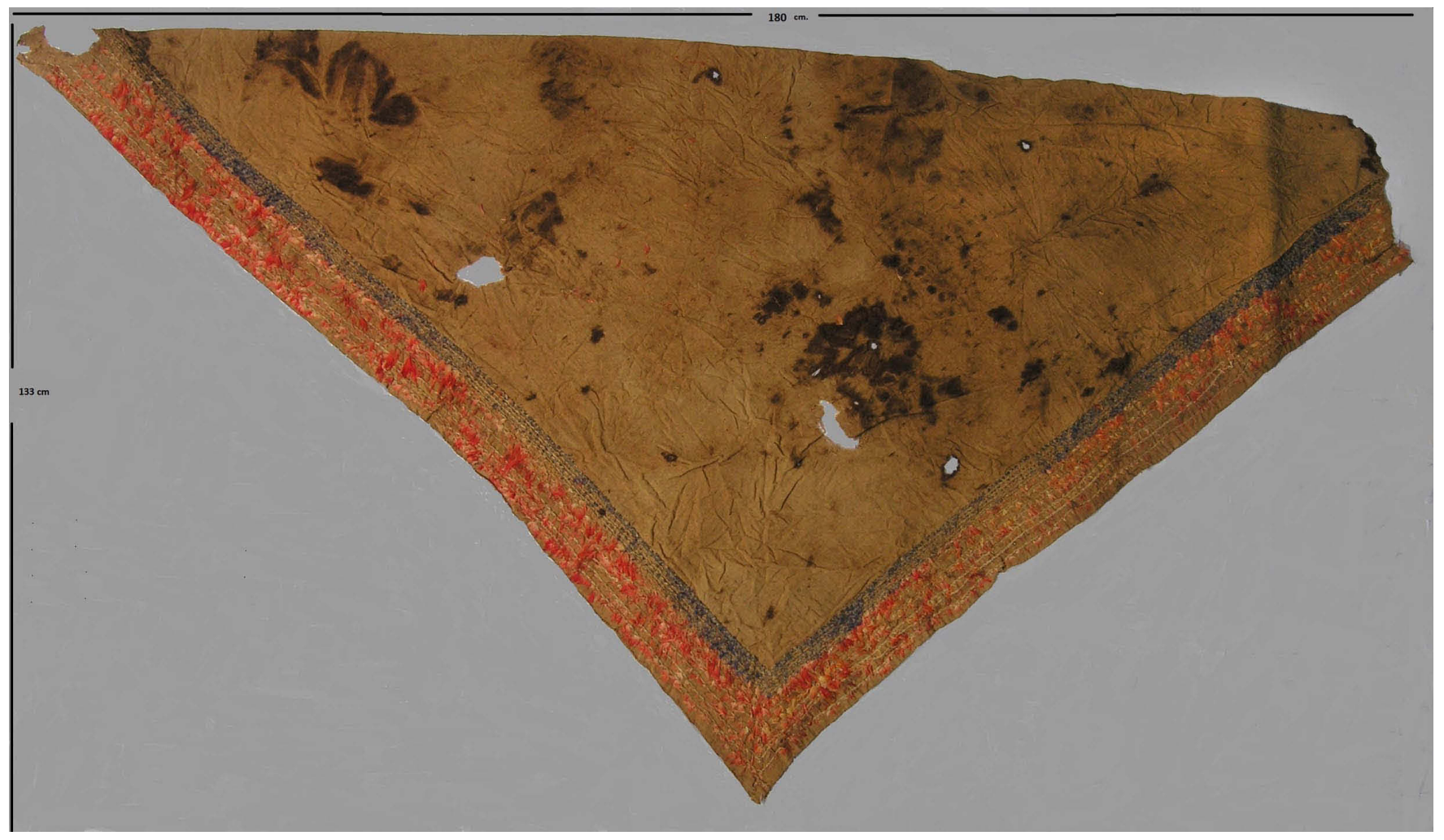

Foto 1: Tejido Triangular con Plumas de Pachacamac. (MSPAC/Foto L. Ch.)

encontrados en Pachacámac como Yschma, se caracterizan principalmente por el uso del algodón, aprovechando los colores naturales de su fibra (beige, crema, blanco y los diferentes tonos de marrón). El algodón también es teñido principalmente de color azul, encontrándose desde los tonos más oscuros hasta los más claros, (algunos variantes de verdes muy suaves). La fibra de algodón sirvió para elaborar paños, mantos para envoltorios, uncus, taparrabos, acsus (prenda femenina). Inclusive el algodón es usado para realizar tejidos con técnica de tapiz ranurado (kelim), característica de la costa central y de los textiles Ychsmas. Las telas pintadas de algodón también se han encontrado en regular cantidad.

El pelo de camélido fue usado preferentemente para la decoración de prendas, mediante técnicas como tramas suplementarias y complementarias (llamado a veces brocado), y tapices; para los cuales se mezclaron las urdimbres de algodón, con las tramas de pelo de camélido y otras veces de algodón. La poca presencia de fibra de camélido en Pachacámac en los períodos tardíos es notoria, si se compara con la abundante presencia de fibra de camélido en tejidos
Chancay, contemporáneos y costeños. La explicación según Feltham y Ángeles (2017) es que la fibra de camélido se conseguía a través del trueque con la etnia de Yauyos, con los cuales Pachacamac no había una buena relación, mientras que para el período inca, las fibras de camélido solo eran utilizadas para las elites. En cuanto a la presencia de plumas como parte decorativa de los tejidos, aparecen en aquellos de estilos foráneos, especialmente los que provenían del norte y de los incas. El trabajo de la aplicación de plumas es atribuido a mitimaes chimús (Feltham y Ángeles 2017). Los tejidos hallados, entre otros objetos, formaron parte de las ofrendas para los ancestros, pero también para el dios Pachacámac (Eeckhout, 2017).

En el catálogo de Pachacámac (Ministerio de Cultura Perú 2012, p. 64) se hace una breve referencia a paños triangulares con aplicaciones de plumas; precisamente se refieren al paño que analizamos, atribuyéndolo al estilo inca, por otro lado el arqueólogo Rommel Ángeles ${ }^{2}$ menciona que existe otro paño similar al que describimos, en la colección de Pachacámac, pero este segundo ejemplar triangular está incompleto y muy fragmentado.

2. Comunicación personal 2018. 


\section{Descripción del paño triangular (punta) ${ }^{3}$}

Como ya hemos mencionado, esta punta tiene la forma de un triángulo obtuso, cuya base mide $180 \mathrm{~cm}$. y cada lado $1.33 \mathrm{~cm}$. Ha sido elaborado de algodón de color beige-cremoso, sin teñir. En los lados más cortos presenta un recamado de plumas distribuidas en dos franjas: una ancha con plumas anaranjadas y una delgada con plumas azules/moradas con visos de tornasol. Las primeras son de mayor tamaño que las segundas. La orientación de las plumas aplicadas en los bordes nos indica la manera correcta de colocar el tejido, pues están dispuestas con los cañones hacia adentro del paño, por lo tanto, el paño debe haberse usado con el ángulo central hacia abajo. (Foto 1)

Material y técnicas de elaboración:

Algodón (gossypium barbadense, sp.) sin teñir, de color beige, ha sido muy notorio y difundido entre las sociedades costeñas prehispánicas. Las fibras utilizadas han sido solamente hiladas en dirección "S", se trata de hilos de un solo cabo, tanto las urdimbres como las tramas. Los hilos son bastante finos, con un hilado mediano, de $65^{\circ}$ a $60^{\circ}$ de inclinación en las urdimbres y tramas. Como resultado es un tejido suave y fino al tacto. El ligamento de confección es tela llana con la variante de 2 (dos) urdimbres $\times 1$ (una) trama, (llamado semibásquet). Es el típico tejido de urdimbres pareadas y tramas unitarias e hilos en dirección "S", cualidades que lo identifican como un tejido de origen chimú (Rowe, 1984).

\section{Confección del paño:}

Por lo general, los paños triangulares son el resultado de tejidos cuadrados que se doblan diagonalmente, como el descrito por Ina VanStan (1965). Menos frecuentes son aquellos que se tejen directamente de esta forma. El tejido triangular de Pachacámac, que estoy analizando, ha sido elaborado mediante dos paños rectangulares, los cuales fueron tejidos en telares de cintura, lo que se deduce de la anchura de cada paño, que es de $65.5 \mathrm{~cm}$. Una vez terminados, fueron sacados del telar y unidos por sus lados longitudinales mediante costuras diagonales pequeñas, realizadas con hilos de algodón de igual color del tejido y dirección del hilado. Los dos paños formaron un tejido cuadrangular, que fue doblado en diagonal por las puntas para obtener una forma triangular (ver esquemas: dibujo 1, 2, 3 y 4). El tejido doblado se cosió y cerró por los bordes con puntadas ligeramente diagonales de hilo de algodón de igual color y torsión que del paño (foto 2). El resultado es un tejido más grueso y más pesado.
El tejido triangular presenta algunos daños como manchas oscuras, arrugas, y pequeños desgarros. Las manchas se deben al contacto de otros materiales orgánicos que han provocado la oxidación de fibras, mientras el análisis de las arrugas no indica que formaran un patrón, pues no se pudo distinguir entre las que posiblemente estaban en el momento del hallazgo y aquellas que se produjeron en el almacenamiento posterior en las cajas de los depósitos del museo. El tratamiento de recuperación del tejido se hizo en el 2009. A pesar de estos problemas, las fibras de algodón en general se encuentran estables, si bien el tejido ha perdido gran cantidad de plumas.

\section{Decoración:}

El recamado de plumas se realizó solamente en una cara del tejido y se hizo una vez que este ya estaba doblado y cerrado en forma de triángulo. Las plumas se han colocado de forma paralela, son ocho sartas de plumas anaranjadas, y siete sartas de plumas azules tornasol. Las plumas anaranjadas están en el orillo de los bordes y en la parte interna las azules tornasol. Las sartas de plumas han sido fijadas desde el borde hacia el campo del tejido, la sujeción de cada sarta o hilera es cubierta por la siguiente sarta que se coloca de forma paralela (foto 3 ).

Las plumas anaranjadas probablemente provengan de guacamayos (ara, $s p{ }^{4}$ ), como el color es muy típico de estas aves, y muchas plumas en el tejido se encuentran incompletas, presentando un color más claro en la base. Christine Giuntini (2012: 63) observa que la base de algunas plumas se presenta de color oscuro pardo, o de color más claro, es indicio que no han sido teñidas y provienen de guacamayos. En el caso de las plumas azul tornasol van desde el azul al violáceo y algunas tienen la base de los cañones más claros. En relación a la procedencia, hay varias posibilidades: pueden ser del ave tangara paradisiaca (O'Neill, 1984:149), o tal vez por la dimensión de las plumas, que es de $2 \mathrm{~cm}$. podrían provenir del cuello o la cabeza de guacamayos. Para definir la proveniencia exacta de estas últimas se requiere un análisis especializado.

\section{Forma de sujeción de las plumas:}

El tipo de nudo o sujeción se realizó con hilos de algodón color crema; algunos hilos tienen hebras combinadas y jaspeadas (cabos beige y marrón o pardo), cuya torsión y grosor se diferencian de los usados en el tejido. Los hilos de sujeción de las plumas son de dos cabos con retorsión en ambas direcciones en $\mathrm{S}$ y retorcidos en $\mathrm{Z}$, y algunos hilados

3. Actualmente los tejidos de esta forma se les denomina punta, puntilla o "chal", y es una prenda femenina, usado para cubrir los hombros o a veces la cabeza.

4. Existen variedades de esta especie que provienen de los bosques tropicales de Sudamérica. 

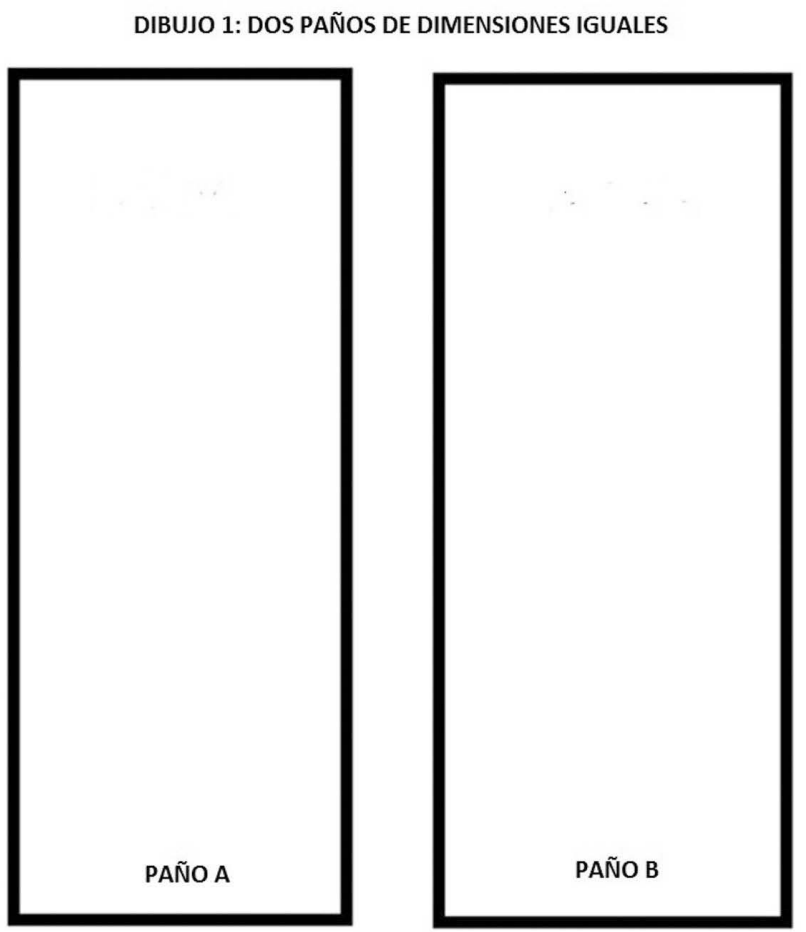

Dibujo: RLCHM

Dibujo esquemático 1: Dos paños iguales de forma rectangular A y B. (Dibujo: L. Ch.)

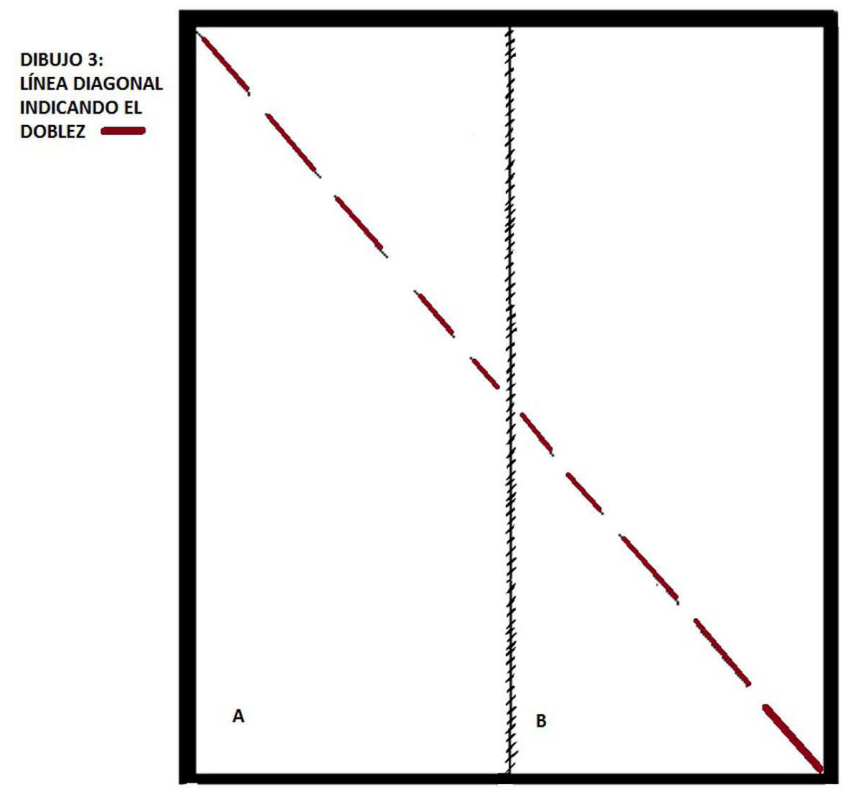

Dibujo esquemático 3: Indica la línea diagonal para doblar el tejido cuadrangular. (Dibujo: L. Ch.)

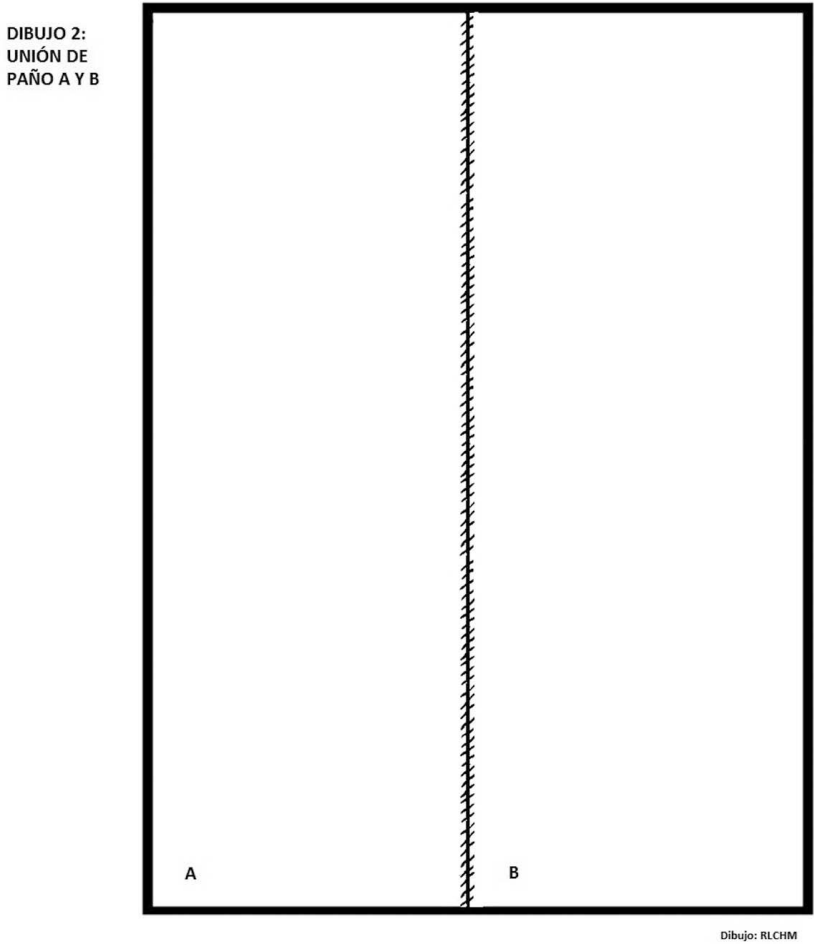

Dibujo esquemático 2: Los paños son unidos por costuras y forman un cuadrángulo. (Dibujo: L. Ch.)

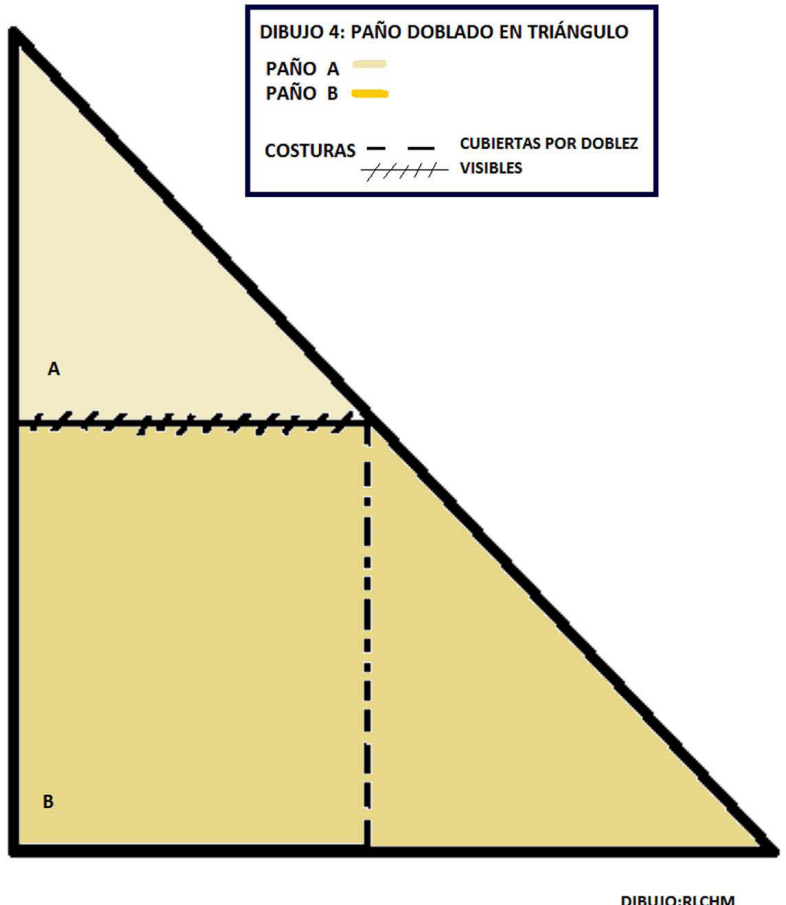

Dibuja esquemático 4: El paño doblado formado un triángulo. (Dibujo: L. Ch.) 


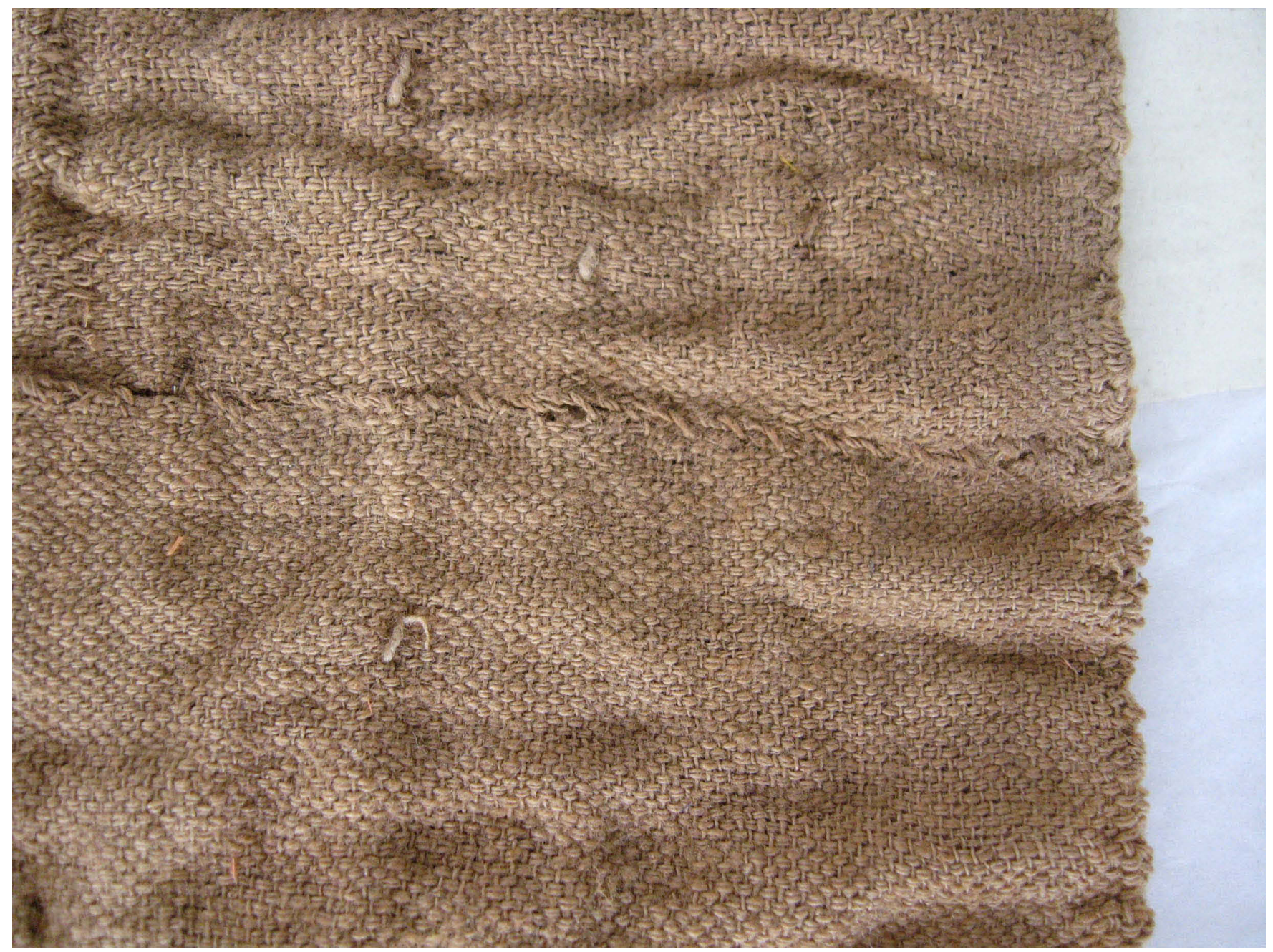

Foto 2: Detalle de las costuras de unión de los paños (a y b). (MSPAC/Foto L. Ch.)

en $\mathrm{Z}$ y doblados en $\mathrm{S}$, siendo este último el tipo de retorsión más frecuente (foto 4). Primero, las plumas han sido dobladas por la base del cañón, luego colocadas sobre un hilo de algodón, y con un segundo hilo o hebra se ha realizado un nudo alrededor del doblez de los cañones de la pluma, asegurando una a una cada pluma con el segundo hilo. Se usa un nudo sencillo, quedando el cañón fijo para formar una sarta o tira de plumas. Luego se ha colocado la sarta de plumas sobre el tejido, y se han fijado mediante puntadas o lazadas envolventes, cada una de ellas de forma continua, se trata de hileras de puntadas muy apretadas. Todas las plumas, tanto las más grandes como las pequeñas, han sido aplicadas de la misma manera (dibujo $\mathrm{n}^{\circ}$ ). En el revés del tejido se puede ver algunas de las puntadas de sujeción de las plumas a la tela, estas puntadas han traspasado los paños doblados, esto nos indica que no se siguió un patrón en cuanto si las plumas debían sujetarse a ambos paños o solo a uno (foto 5 ).
Existen diferentes procedimientos para fijar las plumas en los tejidos, los que han sido descritos y dibujados por varios autores, pero ninguno es completamente igual al que se usó en el paño triangular de Pachacámac. Raoul d'Harcourt (1974: figura 115) describe una forma de fijación de plumas que se parece en las lazadas de sujeción de las plumas a la tela pero es diferente en la forma en que se sujeta cada pluma individualmente. El dibujo publicado por Rowe (1984: 154) describe un tipo de fijación de plumas totalmente diferente. Los dibujos de Giuntini (2012: 96 fig. 65) muestran dos tipos de fijación de dos piezas con plumas respectivamente, pero tampoco se parecen a las del tejido triangular. El dibujo de Al de González en la publicación de Eugene Yacovleff (1933, p. 145, fig. 2) la fijación de las plumas de la parte superior es muy similar a la encontrada en el tejido triangular de Pachacámac, no así las puntadas de fijación, porque el material analizado por Yacovleff no pertenecían a tejidos recamados con plumas. 


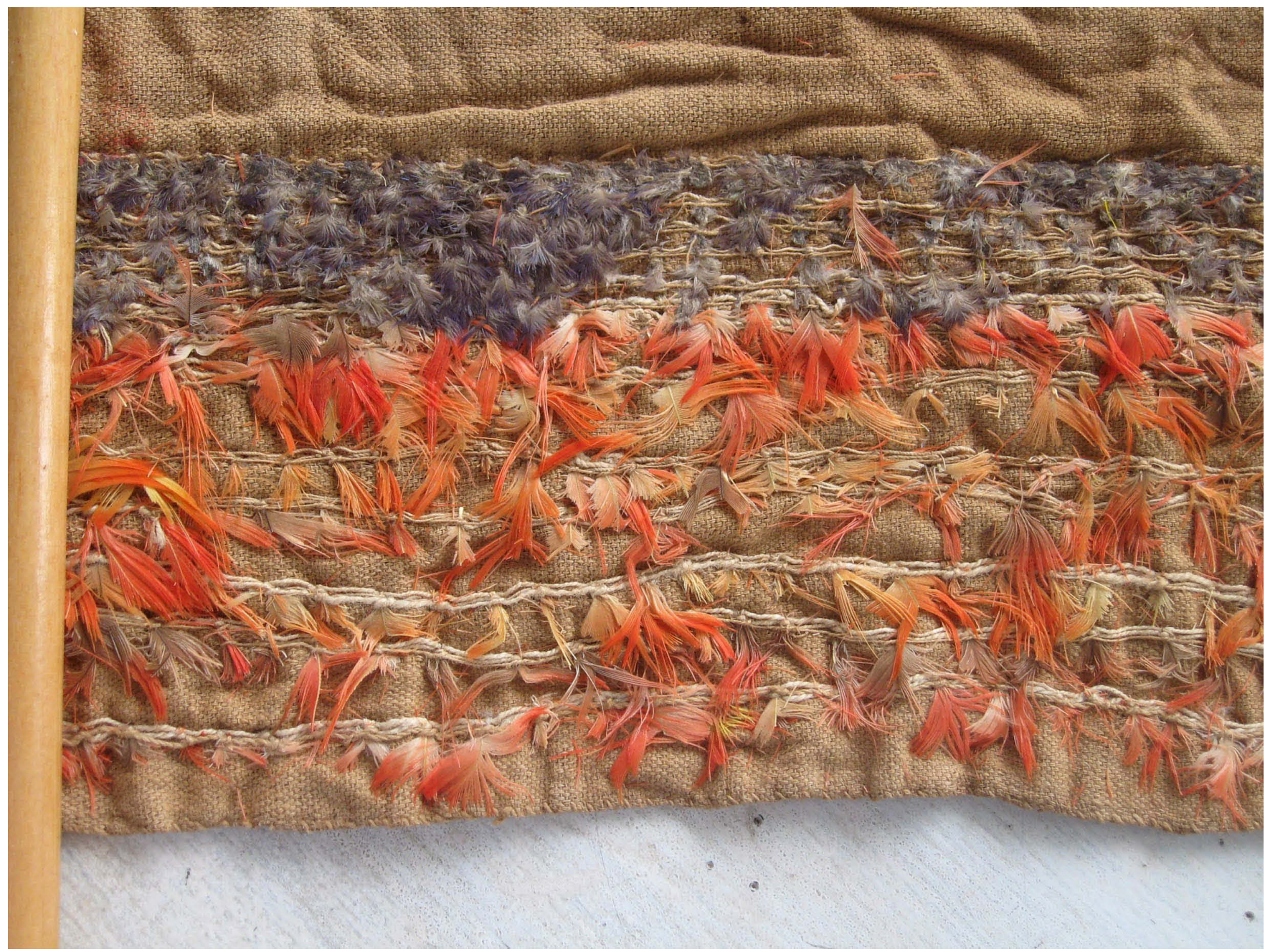

Foto 3: Detalle de la decoración de plumas. (MSPAC/Foto L. Ch.)

Finalmente, la fijación de plumas puede variar (Giuntini, 2012: 95), por lo que no podemos establecer un patrón muy definido, aunque hay algunas coincidencias en la manera como se han fijado las plumas de este paño triangular de Pachacámac y los modelos presentados anteriormente. Se observa además que el trabajo de recamado de plumas en dicho paño no fue muy prolijo porque los hilos de fijación son notorios, aunque quizá esto se deba a la pérdida de plumas ocurrida (foto 6).

\section{Hallazgos de tejidos triangulares}

Tal vez los más antiguos tejidos triangulares prehispánicos reportados son aquellos procedentes de las excavaciones arqueológicas de Paracas realizadas por Julio C. Tello y su equipo (1927 al 1930). Al realizar la clasificación de la vestimenta de Paracas, Rebeca Carrión Cachot (1931) dice que solo se encontraron waras (taparrabos) triangulares en dos de los diez fardos examinados, y que además eran muy pocos en comparación con las waras de forma rectangular. Otro detalle importante es que estos paños triangulares fueron elaborados de un solo paño, es decir que salieron en forma triangular del telar, sin que sea necesario hacerlos a partir de un paño cuadrangular doblado en dos. También en Paracas se encontraron otras formas, por ejemplo los taparrabos en forma de pentágono con un extremo más agudo (Carrión, 1931). Uno de ellos está expuesto en el Museo Regional de Ica y otro en la Sala Paracas del Museo Nacional de Arqueología, Antropología e Historia del Perú (MNAAHP) 5 .

En el fardo 89 de las Necrópolis de Wari Kayan, cementerio en Paracas se encontraron tres piezas triangulares con bordados de estilo Nazca tempano. Un ejemplar mejor conservado es un paño cuadrado doblado diagonalmente, y tiene una banda bordada y cosida en la margen más larga 


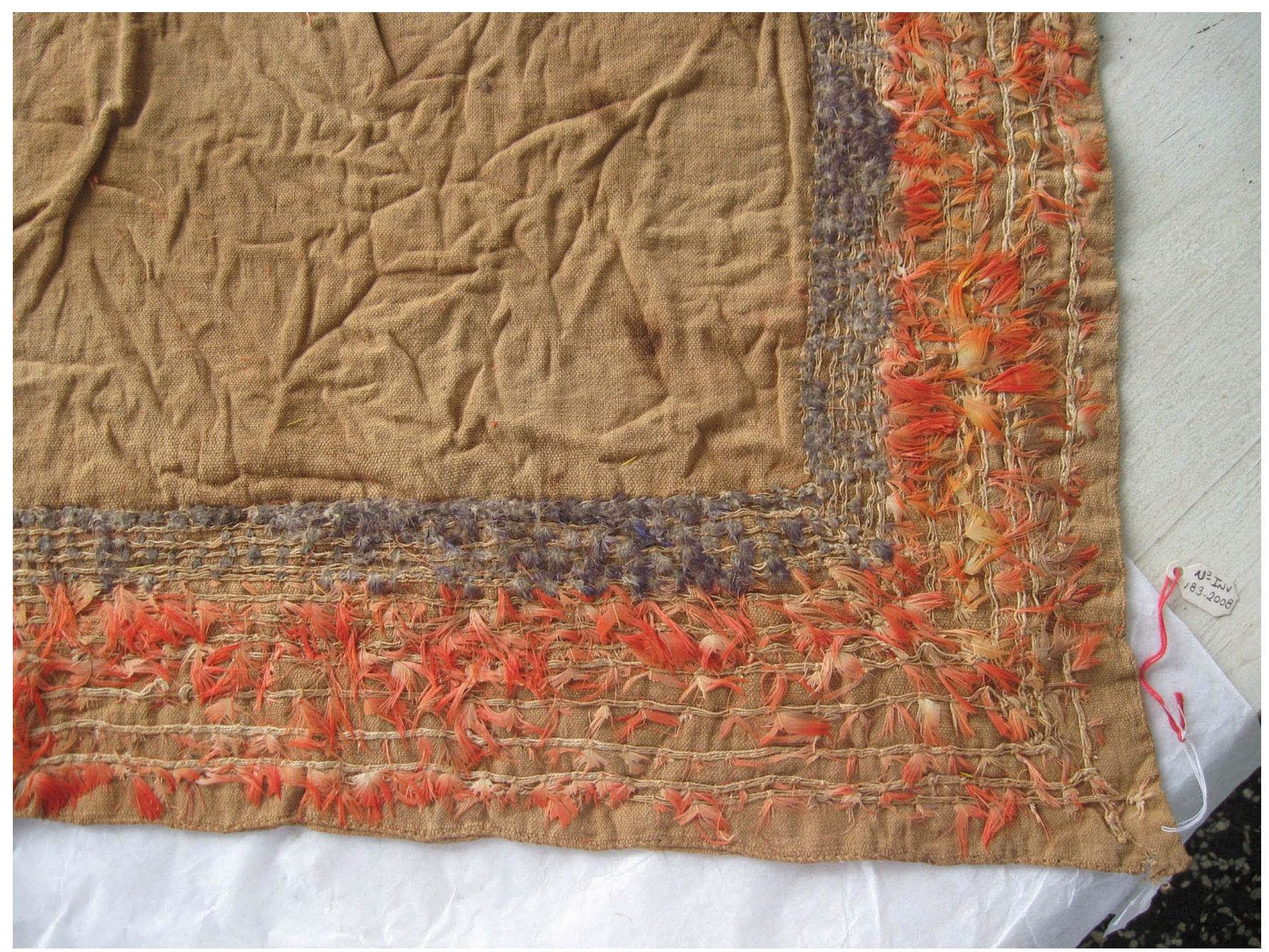

Foto 4: Detalle de fijación de plumas. (MSPAC/Foto L. Ch.)

del doblez, las otras dos prendas son similares, pero están muy fragmentadas. Fueron identificadas como taparrabos por Carrión (1931-49). Luego como posibles taparrabos (Paul 1991-19o) o telas de tocado (Peters, 2017), considerando que eran muy cortas y no había huellas de amarres (Paul, 1991-190).

En el catalogo en línea del MNAAHP aparece un paño de forma triangular ( $\mathrm{N}^{\circ}$ Registro 247917), el cual se elaboró a partir de un tejido cuadrangular, doblado por las puntas para formar un triángulo, tiene borlas largas de color rojo, el ligamento es un tejido llano de tramas y urdimbres parciales, atribuido a la cultura Nazca; este espécimen, cuyas dimensiones son de $26 \mathrm{~cm} \times 59 \mathrm{~cm}$, puede indicar que tal vez sirvió para envolver una ofrenda.

Reiss y Stübel publicaron la ilustración de un paño triangular, encontrado en Ancón, en las excavaciones realizadas entre $1880-1887$. El tejido está formado a partir de un paño cuadrangular, doblado por las puntas, elaborado con

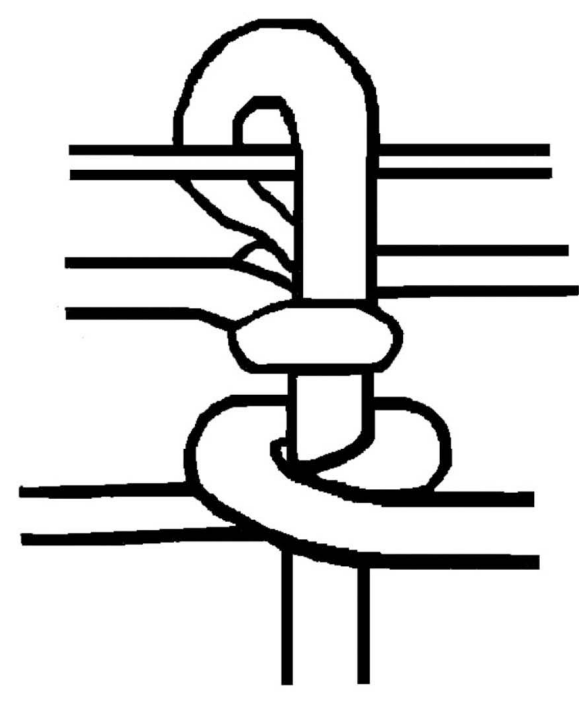

DIBUנO: RLCHM

FIJACION DE PLUMAS DEL TEIDO TRIANGULAR DE PACHACAMAC

Dibujo 5: Esquema de fijación de plumas. (MSPAC/Foto L. Ch.) 


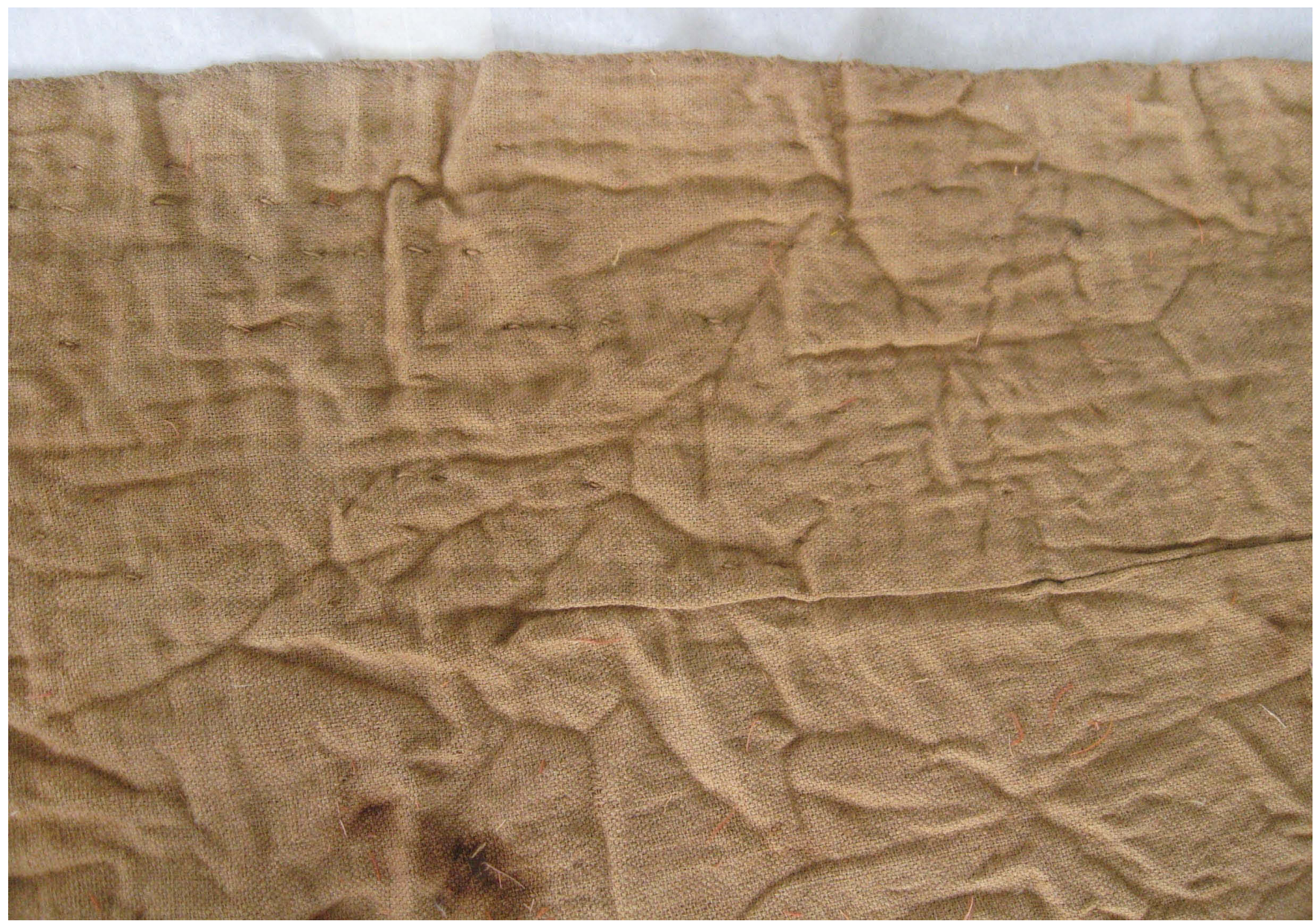

Foto 5: Detalle de costuras posteriores. (MSPAC/Foto L. Ch.)

ligamento de tejido llano ralo (open work) (tramas y urdimbres espaciadas), de algodón de color crema, con líneas más oscuras que forman cuadros, los dos bordes más cortos están decorados con una franja en técnica de tapiz, que termina con tiras en los extremos, lo que sugiere que fue usado para la cabeza, o para envolver y amarrar algún objeto (Reiss y Stübel, vol 2, pp. 146 y 147, lámina 43).

Ina VanStan (1965) reporta un paño triangular muy similar al que se halló en Ancón, pero que proviene de Pachacámac, encontrado en las excavaciones de Max Uhle entre 1896/97. Este tejido triangular, se ha elaborado a partir de un paño cuadrangular de algodón y luego doblado por las puntas. Se tejió mediante la técnica de gasa simple; los lados más cortos tienen bordes rematados por una guardilla elaborada en técnica tapiz ranurada o Kelim (VanStan, 1965: 430, fig. 3 y 4). En el dibujo de la elaboración del paño, se observa que los bordes decorativos de tapiz son añadidos al paño mediante pequeñas puntadas diagonales. Sus dimensiones sugieren que fue usado para la cabeza, a simple vista es similar al paño encontrado en Ancón. En la misma publicación, la autora menciona otro paño, como lo hemos señalado líneas arriba, cuya procedencia es de la costa central, pero no precisa el lugar exacto, este tejido ha sido elaborado de la misma manera que el tejido triangular de plumas de Pachacámac, mediante dos paños rectangulares, unidos y luego doblados, lamentablemente está incompleto, es de algodón de color pardo, con hilos de un solo cabo hilados en "S", y presenta bordes decorados añadidos, en técnica tapiz ranurado, elaborado en fibra de camélido, hilados en $\mathrm{Z}$ y doblados en $\mathrm{S}$ ( $\mathrm{Z} 2 \mathrm{~S}$ ).

En el caso de la Costa Norte específicamente para Moche en la colección del Museo Larco Herrera, hay varias vasijas "huaco retratos" en que los tocados están formados por un paño triangular. Los dos extremos se juntan sobre la coronilla y una punta del triángulo queda detrás. Se ve claramente en los siguientes especímenes: el ML-000036 a, vista c, y el ML-000021 a, vista c, (Catalogo Digital: https://www.museolarco.org/catalogo/ficha.php?id=36, y https://www.museolarco.org/catalogo/ficha.php?id=21).

Al parecer la producción de este tipo de paños fue muy común. Sin embargo, no podemos determinar cómo fueron elaborados. De todos modos, puede haber surgido un indicio 


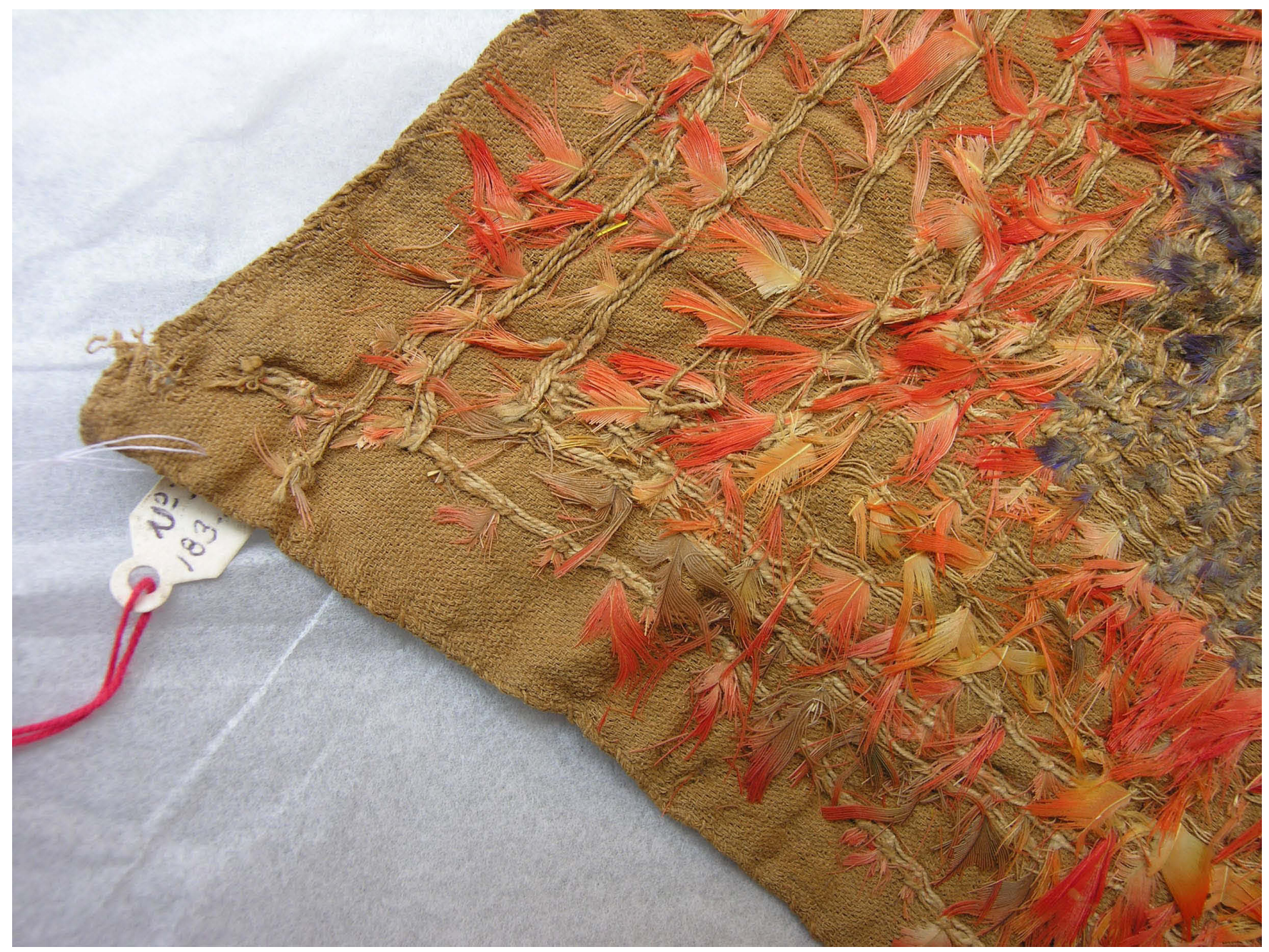

Foto 6: Detalle del paño y costura de fijación de plumas. (MSPAC/Foto L. Ch.)

en las excavaciones en Chornacap, Lambayeque, donde se encontraron contextos funerarios. Entre ellos se descubrieron los restos de una mujer perteneciente a la elite, asociados con objetos de metal, exquisitamente trabajados, y otros materiales ligados a las prácticas shamánicas. Y se le denomina "la Sacerdotisa de Chornacap" (Wester, 2012). En la corona de oro que llevaba dicha "sacerdotisa" aparece la representación de una mujer sentada sobre la media luna sosteniendo un objeto en forma de aspa (X) compuesto por dos triángulos, similar al dibujo del Dr. Zalles (Bjerregaard 2017-34). Se ha planteado que se trata de la representación de la deidad lunar (Wester, 2012: 41, 85), y se la relaciona con la imagen que se encuentra en el "Vaso de Denver" (estudiado por Christopher Donnan), una vasija de estilo Sicán, en que aparece la deidad femenina con similares características encontradas a las que figuran en la corona de la Sacerdotisa de Chornacap (Wester, 2012 85-87). La forma de aspa $\mathrm{o} \times$ que aparece en la corona y el vaso probablemente representa un telar tipo X. Según Feltham y Ángeles (2017) estos telares sirvieron para elaborar pequeños tejidos. Podría suponerse que eventualmente se usaron para confeccionar tejidos triangulares ${ }^{6}$.

En la colección de textiles prehispánicos del Museo Etnográfico de Berlín se encuentran fragmentos de textiles de estilo Sicán/Lambayeque. Lena Bjerregaard reconstruyó hipotéticamente uno de ellos que tenía un lado en punta (2017: 32 - 38) atribuyéndole una forma casi triangular (Bjerregaard, 2017: 35, fig. 5). Otro fragmento, (Bjerregaard 2017: p.106) presenta una figura de dos mujeres de perfil, que portan en la muñeca un hilo con retorsión "S", que acaba en una vara horizontal, que puede ser un huso. Parte de su vestimenta es un paño triangular amarrado alrededor de las caderas, que Bjerregaard ha denominado "paño para ceñir la cintura” (2017: 34). Estos paños triangulares son de Sicán/

6. Lo menciono en relación que artesanalmente se utilizan telares triangulares o bastidores para tejer paños. 


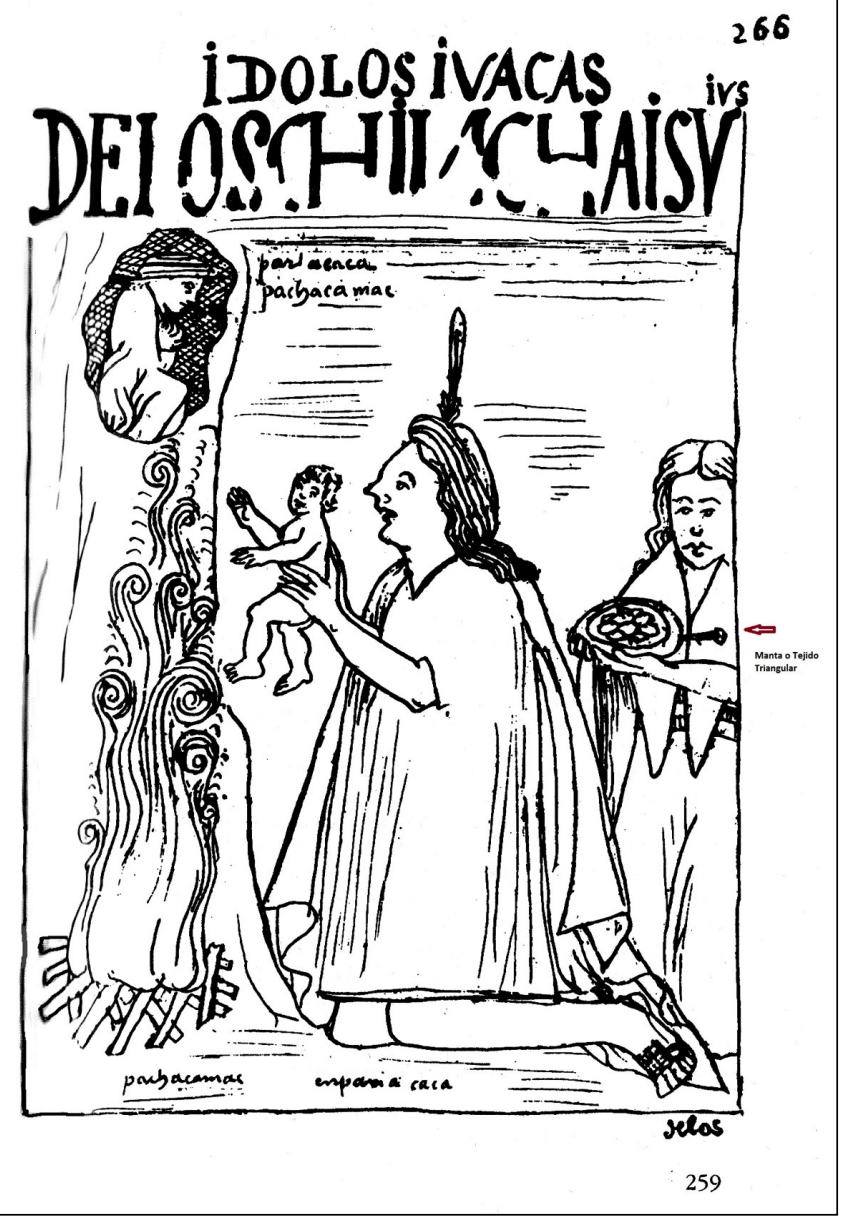

Foto 7: Dibujo por Guaman Poma de mujer con posible manta o tejido triangular. (F. Guaman Poma, 1987 [1615] p. 329)

Lambayeque y se aprecian mejor a través de la iconografía de los fragmentos publicados en el mismo catálogo (2017: 106) 7 . Dichos paños terminan en dos borlas o colgantes en forma de ave que quedan en la parte posterior del cuerpo humano y se ciñen a la cintura con unas cintas que terminan en colgantes con pequeñas cabezas de ave. Estos especímenes podemos relacionarlos con el tercer paño triangular presentado por VanStan (1965, fig. 5) que también tiene decoraciones de borlas.

Para épocas más tardías (Intermedio Tardío y Horizonte Tardío) hay pocos hallazgos de paños triangulares. Existe uno al que se le atribuye el estilo Chancay (Stone, 1994: 248 , fig. 247). Es de algodón en tela llana y presenta bordes tipo tapiz kelim (ranurado), parecido al encontrado en Ancón, con decoración naturalista de peces. Mide $120 \mathrm{~cm}$. $\times 90 \mathrm{~cm}$. incluidas las guardas o bordes decorativos, por lo puede tratarse de un paño cuadrangular doblado por las puntas para formar un triángulo. En la colección el MNAAHP aparece otro paño triangular $\left(\mathrm{N}^{\circ} 231839\right)$ elaborado de tejido llano de algodón, con dos orillos decorados, con técnica de tapiz Kelim (ranurado), y diseños de olas y rostros de serpientes. Presenta los típicos colores rojo y rosado de la cultura Chancay, y es muy parecido al estudiado por Stone (1994), aunque es de mayor dimensión $(120 \mathrm{~cm}$. $\times 116 \mathrm{~cm}$.), es probable que haya sido elaborado también a partir de un tejido cuadrangular. En la ficha del museo está asignado al Intermedio Tardío, y, de acuerdo con las características iconográficas, presenta un estilo marcadamente Chancay. Entre los tejidos de estilo Yschma que han sido estudiados en contextos funerarios no se han reportado formas triangulares, aunque sí hay registro del uso de plumas para algunos textiles (Frame, Vallejo, Ruales y Tosso, 2012: 437 - 478).

La vestimenta de los incas, especialmente la que caracterizaba a la población de la época del Tawantinsuyo, está muy bien representada a través de los dibujos de Guaman Poma de Ayala (1987 Vol. I y II). No aparecen tejidos de forma triangular: las ñañacas o paños para la cabeza, las mantas de los hombres y llicllas de las mujeres, son tejidos rectangulares y/o cuadrangulares. Solamente en una de las ilustraciones (1987 Vol. II: 259, fig. 266), aparece una mujer con una "manta" sobre los hombros, sujeta con un tupu, y parece tratarse de una "manta" triangular (foto 7).

\section{Conclusiones}

1. Los tejidos de forma triangular aparecen en los Andes en todos los períodos. De acuerdo con las indagaciones realizadas, la forma triangular más antigua aparece en el sur, en Paracas, valga mencionar nuevamente los hallazgos de Julio C. Tello y su equipo, y los trabajos sobre indumentaria de Rebeca Carrión Cachot. En este caso fueron usados como taparrabos, aunque cabe la posibilidad que algunos fueran para la cabeza.

2. Por otro lado, se pueden confeccionar paños triangulares siguiendo uno de estos tres procedimientos: 1 . directamente en el telar; 2. A partir de un paño cuadrangular, el cual se dobla diagonalmente por las puntas; y 3. Uniendo dos paños rectangulares para formaran un cuadrado que al ser doblado en diagonal queda en forma triangular; de esta última manera se obtienen tejidos triangulares de mayor dimensión.

7. También se han encontrado fragmentos en Pacatnamu, en las excavaciones de Ran Boytner, quien los menciona en The Pacatnamu Textiles: a study of Identity and Function, Ph.D. Dissertation, University of California, comunicación personal de Lena Bjerregaard, diciembre 2019 . 
3. Los paños triangulares elaborados directamente en los telares aparecen en Paracas y tal vez en Moche y Sicán/ Lambayeque, con el uso del telar en X. En cambio, los paños triangulares elaborados de la segunda forma son los más comunes y se han encontrado desde Intermedio Temprano (Nazca, tal vez en Moche y en Sicán/Lambayeque), pasando por el Horizonte Medio hasta el Intermedio Tardío. Se encuentran especímenes en la costa central (Chancay, Ancón y otros valles). Se han observado que los tejidos triangulares fueron usados para la cabeza, o para envolver ofrendas, también como parte de la indumentaria, en el caso de Sicán/Lambayeque y Paracas.

4. Tenemos algunas piezas elaboradas según el tercer procedimiento: el tejido recamado con plumas de Pachacámac, que hemos analizado; el tercer paño estudiado por Vanstan (1965); los tejidos de Sicán/Lambayeque (que probablemente serían de mayor dimensión, pues se ceñían alrededor del cuerpo).

5. El tejido triangular de plumas de Pachacámac ostenta características que lo identifican con el estilo Chimú: uso de algodón, fibras de un solo cabo, hilado en "S", ligamento de urdimbres pareadas y una sola trama (semibasquet) y la aplicación de plumas.

6. La escasez de tejidos triangulares realizados siguiendo el procedimiento 3 tal vez se deba a que realizarlos implica una planificación diferente a la confección de los paños triangulares realizados siguiendo el segundo procedimiento. Su elaboración debió obedecer a un motivo específico. Según Narváez (2017) la figura del triángulo desempeñó un papel importante en el momento de emplazar ofrendas en los templos incas. Esta disposición se ha encontrado en emplazamientos del norte ocupados por los Incas en territorios de Chimú. Esto puede indicar la importancia simbólica del tejido triangular que analizamos, pues fue encontrado en la "Portada de la Costa", que es la entrada al espacio sagrado de Pachacámac.

7. En el Intermedio tardío la decoración con plumas en muchos textiles de la costa norte, central y sur es frecuente. Muchos autores están de acuerdo con que la utilización de plumas se limitaba a determinadas prendas de algunos personajes importantes, o formaban parte del ajuar funerario, como lo han revelado los hallazgos arqueológicos. Por lo tanto, el tejido triangular presentado probablemente no pudo tener un uso doméstico. Tampoco la presencia de textiles recamados con plumas es extraña en Pachacámac, de alguna manera señala la relación con la región amazónica de donde procedían las plumas. Hasta es posible pensar que se importaran loros y guacamayos.
8. Otra posibilidad es que este tejido, dadas sus dimensiones, pudo servir de manto a alguna mujer, sea cubriéndola desde la cabeza o desde los hombros. Su uso estaría reservado al ámbito ceremonial o como signo de estatus. Es dudoso que se usara ceñido alrededor del cuerpo, porque las plumas se habrían quebrado. El recamado de plumas y el ligamento de su elaboración indican un origen norteño, aunque también cabe la posibilidad de que fuera elaborada en el mismo Pachacámac, siguiendo la tradición norteña.

\section{Referencias citadas:}

Agüero, Carolina

1998 Tradiciones Textiles de Atacama y Tarapacá presentes en Quillagua durante el Período Intermedio Tardío. Boletín del Comité Nacional de Conservación Textil (3): 103 - 128. Santiago.

Bjerregaard, Lena y Huss Torben

2017 Precolumbian Textiles in the Ethnological Museum in Berlin. Zea Books 52 http://digitalcommons.unl.edu/ zeaboook/52

Carrión Cachot, Rebeca

1931 La Indumentaria en la antigua cultura de Paracas. Wira Kocha: Revista Peruana de Estudios Antropológicos Vol. 1. (1): 37 - 86

d'Harcourt, Raoul

1974 Textiles of Ancient Peru and Their Techniques. Mineola New York.

Desrosiers, Sophie

1992 Las Técnicas de tejido ¿tienen un sentido? Una propuesta de lectura de los tejidos andinos. Revista Andina: Tejido Andino Pasado y Presente (1) 10: $7-46$

Eeckhout, Peter

2017 Ofrendas Rituales, Peregrinaciones y Ancestros. Pachacamac el Oráculo en el Horizonte Marino del Sol Poniente. 222 - 236. BCP Lima - Perú

Feltham, Jane, Rommel Ángeles

2017 Los Textiles de Pachacamac. Pachacamac el Oráculo en el Horizonte Marino del Sol Poniente. 251 - 273 BCP Lima - Perú

Frame, Mary, Francisco Vallejo, Mario Ruales y Walter Tosso

2012 Los textiles Ychsma del Horizonte Tardío en el entierro de Armatambo. Arqueología y Sociedad (24): 437-478.

Guamán Poma de Ayala, Felipe

1987 Nueva Crónica y buen gobierno. Crónicas de América $29^{a}$. Ed. John Murra. 3 Volúmenes. España. 
Giuntini, Christine

2012 Techniques and Conservation of Peruvian Feather Mosaics. Peruvian Featherworks. Ar of Precolumbian Era. 89 - 100. Ed. Heidi King. The Metropolitan Museum of Art, New York.

Ministerio de Cultura del Perú

2012 Textiles de Pachacamac. Ministerio de Cultura Perú.

Narváez Vargas, Alfredo

2017 Túcume y Pachacamac entre el Mito y la Arqueología. Pachacamac el Oráculo en el Horizonte Marino del Sol Poniente. 126 - 135. BCP Lima - Perú

Museo Nacional de Arqueología, Antropología e Historia del Perú 2013 Paracas. Ministerio de Cultural. Lima.

O’Neill, John P.

1984 Introduction: Feather Identification. Costumes and Featherwork of the Lords of Chimor: Textiles from Peru's North Coast. 144 - 150. The Textile Museum, Washington D.C.

Paul, Anne

1991 Paracas Necrópolis Bundle 89. Paracas Art \& Architecture. Object \& Context in South Coastal, Peru. Ed. Anne Paul. University Iowa Press.

Peters, Ann

2017 Headdress forms in the Paracas Necrópolis Mortuary Tradition. PreColumbian Textile Conference VII / Jornadas de Textiles PreColombinos VII, ed. Lena Bjerregaard and Ann Peters (Lincoln, NE: Zea Books, 2017). https://digitalcommons.unl.edu/pct7/1/

Ramos Giraldo, Jesús

2011 Santuario de Pachacamac Cien años de arqueología en la Costa Central. Municipalidad Distrital de Lurín. Cultura Andina.
Reiss, Wilhem y Adolph Stübel

1880-1887 The Necropolis of Ancon in Peru: a contribution to Our knowledge of Culture and Industries of the Empire of the Incas. Translated by A.H. Keane, 3 vols. Berlin. Asher and Co.

Rowe, Ann Pollard

1984 Costumes and Featherwork of the Lords of Chimor: Textiles from Peru's North Coast. The Textile Museum, Washington D.C.

1999 Textiles Chimú. Tejidos Milenarios del Peru. Ed. José Antonio Lavalle y Rosario Lavalle. AFP Integra. Lima. $425-479$.

Stone-Miller, Rebeca

1992 To weave for the Sun Ancient Andean Textiles. Thames and Hudson. New York.

VanStan, Ina

1965 A Triangular Scarflike Cloth from Pachacamac, Peru. American Antiquity, vol.30 (4): 428-433. JSTOR, www.jstor.org/stable/277942

1967 Textiles from beneath the Temple of Pachacamac, Peru. Philadelphia: The University Museum, University of Pennsylvania.

Wester La Torre, Carlos

2013 Misterio e Historia en la Cultura Lambayeque La Sacerdotisa de Chornacap. Ministerio de Cultura. Perú.

Yacovleff, Eugene

1933 Arte Plumario entre los antiguos Peruanos. Revista del Museo Nacional 2. (2) 137- 158. Lima. 